\author{
Vijay K Sharma, Subhash C Kapur, Jeevesh Kapur, Arvind K Sinha \\ Division of Neurology, National University Health System, Singapore \\ Yong Loo Lin School of Medicine, National University of Singapore, Singapore
}

\section{Background and aims-}

The preferred neuroimaging in acute ischemic stroke continues to remain $\mathrm{CT}$ based, largely due to the widespread availability, rapid acquisition, and acceptable image quality. However, whether repeated neuroimaging leads to an increased risk of somatic and genetic effects of ionizing radiation remains debatable. Dose tracking software can automatically monitor the radiation received by patients during computerized tomography (CT) based imaging. We evaluated radiation exposure during standard imaging protocols consisting of non-contrast $\mathrm{CT}$ head (NCCT), CT Angiography (CTA) and CT Perfusion (CTP).

Methods: Radiation data was retrieved for various CT based imaging studies, performed for ischemic stroke patients during the year 2015. The volume-weighted CT dose index, dose-length product, scan length, effective dose and whole body integral dose were extracted from dose-tracking software. Radiation doses for brain, skin, eye, thyroid and red bone marrow were extracted.

Results: The radiation data for brain imaging for NCCT ( $\mathrm{n}$ =391), CTA $(n=149)$ and CTP $(n=61)$ were retrieved from the institutional dose tracking system attached to a dedicated CT scanners (Phillips iCT256 with iDose4).

Table 3. Radiation Dose Metrics

\begin{tabular}{|c|c|c|c|c|c|c|}
\hline \multirow[t]{2}{*}{ Variable } & \multicolumn{2}{|l|}{ NCCT } & \multicolumn{2}{|l|}{ CTA } & \multicolumn{2}{|l|}{ CTP } \\
\hline & $\begin{array}{l}\text { Standar } \\
\text { d Dose } \\
(\mathrm{n}=391)\end{array}$ & $\begin{array}{l}\text { Low } \\
\text { Dose } \\
(n=32)\end{array}$ & $\begin{array}{l}\text { Standard } \\
\text { Dose } \\
(n=149)\end{array}$ & $\begin{array}{l}\text { Low Dose } \\
(n=32)\end{array}$ & $\begin{array}{l}\text { Standard } \\
\text { Dose } \\
(n=61)\end{array}$ & $\begin{array}{l}\text { Low Dose } \\
(n=32)\end{array}$ \\
\hline $\begin{array}{l}\text { Mean CTDI } \\
\text { vol in mGy } \\
\text { (SD) }\end{array}$ & $\begin{array}{l}52.4 \\
(0.3)\end{array}$ & $\begin{array}{l}47.4 \\
(0.05)\end{array}$ & $\begin{array}{l}28.7 \\
(8.1)\end{array}$ & $14.7(2.4)$ & $\begin{array}{l}93.0 \\
(0.9)\end{array}$ & $\begin{array}{l}72.0 \\
(1.4)\end{array}$ \\
\hline $\begin{array}{l}\text { Mean DLP in } \\
\text { mGy.cm (SD) }\end{array}$ & $\begin{array}{l}1061.9 \\
(272.8)\end{array}$ & $\begin{array}{l}972.9 \\
(260.2) \\
\end{array}$ & $\begin{array}{l}1415.5 \\
(471.1) \\
\end{array}$ & $\begin{array}{l}584.6 \\
(281.6) \\
\end{array}$ & $\begin{array}{l}1916.2 \\
(339.7) \\
\end{array}$ & $\begin{array}{l}995.1 \\
(233.2) \\
\end{array}$ \\
\hline $\begin{array}{l}\text { Mean ED } \\
\text { ICRP } 103 \text { in } \\
\text { mGy (SD) }\end{array}$ & $\begin{array}{l}2.6 \\
(0.7)\end{array}$ & $\begin{array}{l}2.4 \\
(0.6)\end{array}$ & $\begin{array}{l}4.5 \\
(2.5)\end{array}$ & $\begin{array}{l}1.6 \\
(0.7)\end{array}$ & $\begin{array}{l}4.5 \\
(0.9)\end{array}$ & $\begin{array}{l}2.2 \\
(0.5)\end{array}$ \\
\hline $\begin{array}{l}\text { Whole Body } \\
\text { integral in } \\
\text { mGy (SD) }\end{array}$ & $\begin{array}{l}0.4 \\
(0.1)\end{array}$ & $\begin{array}{l}0.39 \\
(0.1)\end{array}$ & $\begin{array}{l}0.6 \\
(0.2)\end{array}$ & $\begin{array}{l}0.2 \\
(0.02)\end{array}$ & $\begin{array}{l}0.8 \\
(0.14)\end{array}$ & $\begin{array}{l}0.4 \\
(0.09)\end{array}$ \\
\hline
\end{tabular}

*All differences are statistically significant $(p<0.001)$
Table1. Stroke Protocol Scanning Parameters.

\begin{tabular}{|l|c|c|c|c|c|c|}
\hline Variable & \multicolumn{2}{|c|}{ NCCT } & \multicolumn{2}{c|}{ CTA } & \multicolumn{2}{c|}{ CTP } \\
\hline & $\begin{array}{c}\text { Standard } \\
\text { Dose }\end{array}$ & $\begin{array}{c}\text { Low } \\
\text { Dose }\end{array}$ & $\begin{array}{c}\text { Standard } \\
\text { Dose }\end{array}$ & $\begin{array}{c}\text { Low } \\
\text { Dose }\end{array}$ & $\begin{array}{c}\text { Standard } \\
\text { Dose }\end{array}$ & $\begin{array}{c}\text { Low } \\
\text { Dose }\end{array}$ \\
\hline Scan type & Helical & Helical & Helical & Helical & Axial & Axial \\
\hline $\begin{array}{l}\text { Detector } \\
\text { Cofiguration }\end{array}$ & $64 \times 0.625$ & $64 \times$ & $128 \times$ & $128 \times$ & $64 \times 0.625$ & $64 X$ \\
\hline Pitch & 0.4 & 0.4 & 0.4 & 0.4 & - & 0.625 \\
\hline FOV (mm) & 250 & 250 & 260 & 250 & 250 & 250 \\
\hline kVp & 120 & 120 & 120 & 100 & 80 & 80 \\
\hline mAs/SLICE & 220 & 280 & 300 & 200 & 150 & 100 \\
\hline $\begin{array}{l}\text { Rotation Time } \\
\text { (sec) }\end{array}$ & 0.5 & 0.5 & 0.5 & 0.5 & 0.5 & 0.5 \\
\hline $\begin{array}{l}\text { Section } \\
\text { thickness (mm) }\end{array}$ & 3 & 3 & 1 & 1 & 5 & 5 \\
\hline $\begin{array}{l}\text { CTDI vol } \\
\text { (mGy) }\end{array}$ & 54.4 & 44.4 & 38.3 & 15.6 & 120 & 72 \\
\hline
\end{tabular}

\section{Table 2. Patient Metrics}

\begin{tabular}{|c|c|c|c|c|c|c|c|c|c|}
\hline Variable & NCCT & & & CTA & & & CTP & & \\
\hline & $\begin{array}{l}\text { Standar } \\
\text { d Dose } \\
(n=391)\end{array}$ & $\begin{array}{l}\text { Low } \\
\text { Dose } \\
(n=32)\end{array}$ & \begin{tabular}{|l|}
$P$ \\
value
\end{tabular} & $\begin{array}{l}\text { Standar } \\
\text { d Dose } \\
(n=149)\end{array}$ & $\begin{array}{l}\text { Low } \\
\text { Dose } \\
(n=32)\end{array}$ & $\begin{array}{l}P \\
\text { value }\end{array}$ & $\begin{array}{l}\text { Standar } \\
\text { d Dose } \\
(n=61)\end{array}$ & $\begin{array}{l}\text { Low } \\
\text { Dose } \\
(n=32)\end{array}$ & $\begin{array}{l}P \\
\text { value }\end{array}$ \\
\hline $\begin{array}{l}\text { Mean } \\
\text { Age in } \\
\text { years } \\
( \pm S D)\end{array}$ & $62(17)$ & \begin{tabular}{|l|}
63 \\
$(17)$
\end{tabular} & 0.098 & $59(14)$ & $63(17)$ & 0.087 & $60(17)$ & $63(17)$ & 0.082 \\
\hline $\begin{array}{l}\text { Mean } \\
\text { Skull } \\
\text { Diameter } \\
\text { in mm } \\
( \pm S D)\end{array}$ & $171.4(6.9)$ & $\begin{array}{l}171.3 \\
(7.3)\end{array}$ & 0.092 & $\begin{array}{l}186.8 \\
(17.6)\end{array}$ & $\begin{array}{l}186.3 \\
(24.3)\end{array}$ & 0.074 & $\begin{array}{l}172.7 \\
(23.1)\end{array}$ & $\begin{array}{l}172.6 \\
(30.1)\end{array}$ & 0.95 \\
\hline $\begin{array}{l}\text { Water } \\
\text { Equivale } \\
\mathrm{nt} \\
\text { Diameter } \\
\text { in mm }\end{array}$ & $164.9(7.9)$ & $\begin{array}{l}164.3 \\
(7.9)\end{array}$ & 0.022 & $\begin{array}{l}186.8 \\
(17.6)\end{array}$ & $\begin{array}{l}192.5 \\
(38.5)\end{array}$ & 0.067 & 172.4 & 176.2 & 0.068 \\
\hline $\begin{array}{l}\text { Scan } \\
\text { length in } \\
\mathrm{mm}\end{array}$ & $\begin{array}{l}202.8 \\
(52.1)\end{array}$ & \begin{tabular}{|l|}
204.5 \\
$(54.8)$
\end{tabular} & 0.091 & $\begin{array}{l}558.1 \\
(364.9)\end{array}$ & $\begin{array}{l}395.1 \\
(320.8)\end{array}$ & $<0.001$ & $\begin{array}{l}2114.4 \\
(278.1)\end{array}$ & $\begin{array}{l}1912.4 \\
(418.9)\end{array}$ & $<0.001$ \\
\hline
\end{tabular}

Table 4. Radiation Dose to Various Sensitive Organs

\begin{tabular}{|c|c|c|c|c|c|c|}
\hline Variable & NCCT & & CTA & & CTP & \\
\hline & $\begin{array}{l}\text { Standard } \\
\text { Dose }(n=391)\end{array}$ & $\begin{array}{l}\text { Low } \\
\text { Dose } \\
(n=32)\end{array}$ & $\begin{array}{l}\text { Standard } \\
\text { Dose } \\
(n=149)\end{array}$ & $\begin{array}{l}\text { Low } \\
\text { Dose } \\
(n=32)\end{array}$ & $\begin{array}{l}\text { Standard } \\
\text { Dose } \\
(n=61)\end{array}$ & $\begin{array}{l}\text { Low Dose } \\
(n=32)\end{array}$ \\
\hline $\begin{array}{l}\text { Mean Brain dose in mGy } \\
\text { (SD) }\end{array}$ & $\begin{array}{l}57.7 \\
(12.1)\end{array}$ & $\begin{array}{l}43.1 \\
(9.1) \\
\end{array}$ & $\begin{array}{l}101.8 \\
(17.8)\end{array}$ & $\begin{array}{l}20.7 \\
(5.7) \\
\end{array}$ & $\begin{array}{l}82.1 \\
(3.2)\end{array}$ & $\begin{array}{l}55.7 \\
(4.5) \\
\end{array}$ \\
\hline $\begin{array}{l}\text { Mean Skin dose in mGy } \\
\text { (SD) }\end{array}$ & $\begin{array}{l}8.5 \\
(3.1)\end{array}$ & $\begin{array}{l}5.8 \\
(1.6)\end{array}$ & $\begin{array}{l}11.6 \\
(2.2)\end{array}$ & $\begin{array}{l}2.6 \\
(1.8)\end{array}$ & $\begin{array}{l}11.2 \\
(1.7)\end{array}$ & $\begin{array}{l}6.1 \\
(1.5)\end{array}$ \\
\hline $\begin{array}{l}\text { Mean Red Bone Marrow } \\
\text { dose in mGy (SD) }\end{array}$ & $\begin{array}{l}7.4 \\
(2.9) \\
\end{array}$ & $\begin{array}{l}5.2 \\
(1.5) \\
\end{array}$ & $\begin{array}{l}9.7 \\
(2.9) \\
\end{array}$ & $\begin{array}{l}2.6 \\
(1.5) \\
\end{array}$ & $\begin{array}{l}8.7 \\
(0.9) \\
\end{array}$ & $\begin{array}{l}4.7 \\
(1.3) \\
\end{array}$ \\
\hline $\begin{array}{l}\text { Mean Eye dose in mGy } \\
\text { (SD) }\end{array}$ & $\begin{array}{l}83.4 \\
(17.4) \\
\end{array}$ & $\begin{array}{l}63.6 \\
(17.8) \\
\end{array}$ & $\begin{array}{l}159.1 \\
(26.7) \\
\end{array}$ & $\begin{array}{l}33.6 \\
(11.7) \\
\end{array}$ & $\begin{array}{l}152 \\
(3.2) \\
\end{array}$ & $\begin{array}{l}100.3 \\
(24.6) \\
\end{array}$ \\
\hline $\begin{array}{l}\text { Mean Thyroid dose in } \\
\text { mGy (SD) }\end{array}$ & $\begin{array}{l}26.6 \\
(9.4) \\
\end{array}$ & $\begin{array}{l}8.7 \\
(3.8) \\
\end{array}$ & $\begin{array}{l}13.4 \\
(5.8) \\
\end{array}$ & $\begin{array}{l}11.9 \\
(3.9) \\
\end{array}$ & $\begin{array}{l}5.1 \\
(0.9) \\
\end{array}$ & $\begin{array}{l}3.3 \\
(0.8) \\
\end{array}$ \\
\hline
\end{tabular}

*All differences are statistically significant $(p<0.001)$

\title{
Conclusions:
}

1. Radiation exposures of sensitive organs are within acceptable limits with standard neuroimaging protocols for AIS.

2. Lower-dose CT imaging protocols could reduce the radiation doses to the radiosensitive organs without appreciable deterioration in image quality. 